\title{
Prácticas educativas en salud en la escuela: una revisión integrativa
}

\section{Educational practices in school health: an integrative review Práticas educativas em saúde escolar: uma revisão integrativa}

\author{
Tatiane Marinz de Souza Luquez ${ }^{1}$, Vera Maria Sabóiaª \\ ${ }^{1}$ Enfermeira, Especialidade em Saúde Coletiva e Gestão em Saúde, Mestranda, Programa de Pós-Graduação em Enfermagem, \\ Universidade Federal Fluminense.E-mail: tatianemarinz@yahoo.com.br \\ ${ }^{2}$ Enfermeira, Professora Titular da Disciplina de Fundamentos de Enfermagem da escoal de Enfermagem da Universidade \\ Federal Fluminense. E-mail: verasaboia@uol.com.br \\ Cómo citar este artículo en edición digital: Luquez, T.M.S., \& Sabóia, V.Ma.. (2017). Prácticas educativas en salud en la escue- \\ la: una revisión integrativa. Cultura de los Cuidados (Edición digital) 21,47. \\ Recuperado de http://dx.doi.org/10.7184/cuid.2017.47.15 \\ Correspondencia: Tatiane Marinz de Souza Luquez. Rua Cornélio José Pereira, no 61. São Gonçalo. (Rio de Janeiro/Brasil). \\ Correo electrónico: tatianemarinz@yahoo.com.br. \\ Recibido: 20/07/2016; Aceptado: 21/12/2016
}

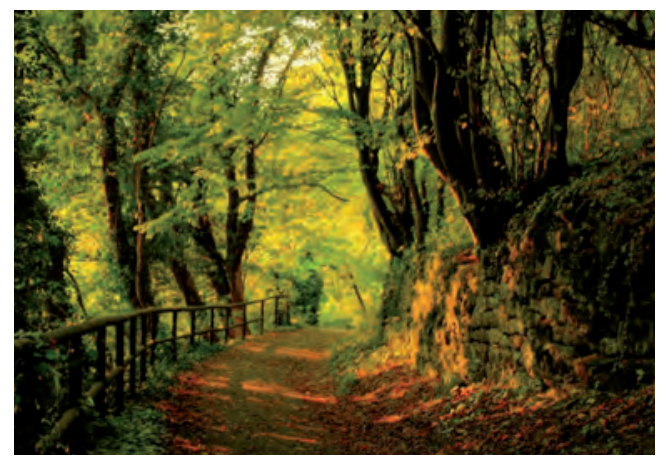

\section{ABSTRACT}

The school is an important stage in participatory construction health reality, encouraging reflection and development of educational health promotion practices. The objective is to know the approaches that have guided the education of health promotion practices at school. This is an integrative review, to search in the databases LILACS, SCIELO, MEDLINE and PubMed. Guiding question: "What educational aspects have guided research on health education practices in scientific schools published in journals in the health area in the period 2005-2014?" Were included articles published in Portuguese, English and Span- ish; in full; which reflect the theme, published and indexed in databases surveyed from 2005 to 2014. We selected 15 articles and analyzed in two categories: The training of teachers and the impact on the practice of school health; and Educational aspects present in the practice of school health. The practice of school health, still needs improvement in the actions of educators in the school health program. One can understand the need for new interventions appropriate to the reality of the students, considering socioeconomic and cultural aspects, environmental influences, behavioral and personal characteristics that are aimed at improving the quality of life and health of the school population.

Keywords: School health, health education, health promotion, child health.

\section{RESUMEN}

La escuela es una etapa importante en la realidad participativa de construcción de la salud, favoreciendo la reflexión y el desarrollo de las prácticas de promoción de la salud educativos. El objetivo es conocer los criterios que han guiado la educación de las prácticas de promo- 
ción de la salud en la escuela. Se trata de una revisión integradora, para buscar en las bases de datos LILACS, SciELO, MEDLINE y PubMed. pregunta guía: ¿"Qué aspectos educativos han guiado la investigación sobre las prácticas de educación sanitaria en las escuelas científicos publicados en revistas en el área de la salud en el período 2005-2014?”, fueron publicados los artículos incluidos en portugués, inglés y español; en su totalidad; que reflejan el tema, publicados e indexados en bases de datos de la encuesta de 2005 a 2014. Se seleccionaron 15 artículos y se analizó en dos categorías: La formación de los profesores y el impacto en la práctica de la salud en la escuela; y los aspectos educativos presentes en la práctica de la salud en la escuela. La práctica de la salud en la escuela, todavía necesita mejoras en las acciones de los educadores en el programa de salud escolar. Uno puede entender la necesidad de nuevas intervenciones apropiadas a la realidad de los alumnos, teniendo en cuenta los aspectos socioeconómicos y culturales, influencias ambientales, características de comportamiento y personales que tienen por objeto mejorar la calidad de vida y la salud de la población escolar.

Palabras clave: Salud escolar, educación para la salud, promoción de la salud, salud infantil.

\section{RESUMO}

A escola é um cenário importante na construção participativa de realidade em saúde, favorecendo reflexão e desenvolvimento de práticas educativas de promoção da saúde. Objetiva-se conhecer as abordagens que tem orientado as práticas educativas de promoção da saúde na escola. Trata-se de uma revisão integrativa, com busca nas bases de dados LILACS, SCIELO, MEDLINE e PUBMED.
Questão norteadora: "Que vertentes educacionais têm orientado as pesquisas sobre as práticas de educação em saúde nas escolas publicadas em periódicos científicos da área de saúde no período de 2005 a 2014?” Foram incluídos artigos publicados em português, inglês e espanhol; na íntegra; que retratassem a temática, publicados e indexados nos bancos de dados no período de 2005 a 2014. Foram selecionados 15 artigos e analisados em duas categorias: A formação dos educadores e a repercussão na prática da saúde escolar; e As vertentes educacionais presentes na prática da saúde escolar. A prática de saúde escolar, ainda necessita de aprimoramento nas ações dos educadores no programa de saúde escolar. Compreende-se a necessidade de novas intervenções adequadas à realidade dos educandos, considerando aspectos socioeconômicos e culturais, influências ambientais, características comportamentais e pessoais visando melhorias na qualidade de vida e saúde da população escolar.

Palavras chave: Saúde escolar, educação em saúde, promoção da saúde, saúde da criança.

\section{INTRODUÇÃO}

A comunidade escolar é um espaço de cuidar em saúde de grande viabilidade para a concretização de ações de promoção da saúde voltadas para o fortalecimento das capacidades dos indivíduos que frequentam o ambiente escolar, a tomada de decisões favoráveis à sua saúde, a criação de ambientes saudáveis e a consolidação de uma política intersetorial voltada para a qualidade de vida (Balbino, 2010).

Há registros de atividades de saúde escolar no mundo desde o século XVIII. No Brasil, a saúde escolar também é uma prática antiga, com registros desde 1850 . No entanto, as práticas realizadas eram fundamentadas na lógica 
biomédica e no policiamento de doenças. Havia um movimento em defesa da promoção de medidas sanitárias de higiene, lutando contra as doenças infecciosas, produzidas pelas más condições de vida, tentando-se assim, erradicar focos infecciosos. A visão higienista surgiu a partir de mudanças na percepção da infância que passou a ser considerada essencial no desenvolvimento individual e social. Além disso, existia interesse na classe trabalhadora visando cidadãos saudáveis e produtivos. Assim, a escola passou a ser percebida como um lugar privilegiado para desenvolver atividades higienistas e preventivas (Maté, Maté, 2015).

Com a evolução técnico-científica ocorrida no século XX, aos poucos se iniciou um movimento de transformação do discurso biomédico nas escolas, para um discurso progressista onde a escola é considerada como um veículo promotor de saúde, por meio de um trabalho articulado entre educação, saúde e a sociedade (Figueiredo, Machado, Abreu, 2010).

As necessidades e os problemas de saúde devem ser identificados a fim de definir estratégias de promoção à saúde no espaço escolar com enfoque integral. Porém, os profissionais que atuam nesse cenário continuamente entendem a educação em saúde na escola como sendo intervenções pontuais, mantendo assim a relação entre saúde e educação focada no modelo biomédico e no controle de doenças, fazendo com que essa prática educativa apresente-se pouco eficaz no sentido de promover reorientações (Figueiredo, Machado, Abreu, 2010).

Nesse contexto, a educação em saúde pautada nas necessidades desse grupo populacional específico, reafirma a importância de uma visão problematizadora, que leva em consideração o contexto e saberes do educando, a troca de conhecimento e a construção participativa de ideias e reflexões.
Dessa forma, é fundamental conhecer as abordagens educacionais adotadas na prática da saúde escolar, assim como as dificuldades para o seu desenvolvimento, com vistas a despertar outros estudos e motivar ações educativas em saúde com escolares, e o planejamento e execução de ações, numa visão reflexiva e crítica, que promova mudanças sociais. Para tanto, objetiva-se com este estudo conhecer as vertentes educacionais que tem orientado as práticas de educação em saúde nas escolas publicadas em periódicos científicos da área de saúde, no período de 2005 a 2014.

\section{MÉTODO}

A presente pesquisa apresenta como método a revisão integrativa, a qual inclui a análise de pesquisas relevantes que dão suporte para a tomada de decisão relativa à temática. É um método de pesquisa que permite a busca, a avaliação crítica e a síntese das evidências disponíveis do tema investigado, combinando dados da literatura teórica e empírica, os conceitos e os objetivos, sendo o seu produto final o estado atual do conhecimento do tema investigado (Mendes, Silveira, Galvão, 2008; Martins, Brito, 2013).

Para a realização da revisão integrativa é preciso percorrer seis etapas distintas: identificação do tema e questão de pesquisa; estabelecimento de critérios de inclusão e exclusão/ busca na literatura ou amostragem; categorização dos estudos/ definição das informações a serem extraídas; avaliação dos estudos; interpretação dos resultados; e apresentação da revisão/síntese do conhecimento (Mendes, Silveira, Galvão, 2008). Desta maneira, para guiar a pesquisa, formulou-se a seguinte questão: Que vertentes educacionais têm orientado as pesquisas sobre as práticas de educação em saúde nas escolas publicadas em periódi- 
cos científicos da área de saúde no período de 2005 a 2014 ?

Os critérios de inclusão utilizados foram artigos publicados em português, inglês e espanhol; artigos na íntegra; artigos que retratassem a temática sobre as práticas educativas em saúde na escola e artigos publicados e indexados nos bancos de dados pesquisados no período de 2005 a 2014. Os Critérios de exclusão foram artigos publicados que se repetiram nas bases de dados; artigos em outras línguas; outras obras, como teses, dissertações, capítulos de livros; e artigos que não estavam publicados na íntegra.

Foram consultadas as seguintes bases de dados: LILACS (Literatura Latino Americana e do Caribe em Ciências da Saúde), SCIELO (Scientific Eletronic Library Online), MEDLINE (United States National Library of Medicine) e PUBMED em janeiro de 2015.

O uso dos descritores: saúde da criança, saúde escolar, educação em saúde e promoção da saúde ocorreu através de combinações e a utilização do operador booleano AND. Vale ressaltar que quando foi feita a pesquisa da base MEDLINE, foi gerado uma mensagem informando que nenhum resultado havia sido encontrado e que deveria se redirecionar para o PUBMED.

Para realizar a apreensão dos conhecimentos produzidos nas publicações, foram realizadas leituras aprofundadas para reflexões críticas quanto às seguintes informações: título; ano/fonte; objetivo; metodologia; resultados; conclusões/ recomendações do (s) autor(es) e base de dados.

\section{RESULTADOS}

Foram selecionados no total final para a realização do estudo, 15 (quinze) artigos que se enquadraram nos critérios de seleção, sendo encontrado nas bases de dados: 33,34 \% (5 artigos) na LILACS, 33,34 \% (5 artigos) na SCIELO e 33,34 \% (5 artigos) no PUBMED.

Em relação aos idiomas, do total dos artigos selecionados, 60\% (09 artigos) são em português, 33,34\% (05 artigos) em inglês e 6,67 $\%$ (01 artigo) em espanhol. Quanto ao ano de publicação dos artigos selecionados nas bases de dados pesquisadas de 2005 a 2014, em 2014 foi obtido um percentual de 6,67\% (01 artigo), em 2013 26,67\% (04 artigos), em 2012 6,67\% (01 artigo), em 2011 20\% (03 artigos), em 2010 13,33\% (02 artigos), em 2009 6,67\% (01 artigo), em 2008 6,67\% (01 artigo), em 2007 6,67\% (01 artigo), em 2006 0\% (nenhum artigo), e em 2005 6,67\% (01 artigo).

A Tabela 1 caracteriza os artigos acerca do tipo, do delineamento e da natureza do estudo dos 15 (quinze) artigos selecionados como amostra final.

Niterói - RJ, 2015

\begin{tabular}{|c|c|c|}
\hline TIPO DO ESTUDO & $\mathbf{N}$ & $\%$ \\
\hline Pesquisa de campo & 03 & 20 \\
\hline Descritivo-exploratório & 06 & 40 \\
\hline Revisão de literatura & 01 & 6,67 \\
\hline Pesquisa participante & 01 & 6,67 \\
\hline Descritivo & 02 & 13,33 \\
\hline Observacional & 02 & 13,33 \\
\hline NATUREZA & $\mathbf{N}$ & $\%$ \\
\hline Qualitativo & 08 & 53,33 \\
\hline Quantitativo & 03 & 20 \\
\hline Quanti-Qualitativo & 04 & 26,67 \\
\hline DELINEAMENTO DO ESTUDO & $\mathbf{N}$ & $\%$ \\
\hline Bibliográfico & 01 & 6,67 \\
\hline Longitudinal & 03 & 20 \\
\hline Transversal & 01 & 6,67 \\
\hline Relato de experiência & 03 & 20 \\
\hline Não especificado & 07 & 46,67 \\
\hline
\end{tabular}

De acordo com a tabela 1, verificar-se o predomínio de $40 \%$ de estudos do tipo descritivo-exploratório (06 artigos). Os tipos menos utilizados foram a pesquisa participante com $6,67 \%$ (01 artigo) e 6,67\% de revisão de literatura (01 artigo). Quanto à natureza dos es- 
tudos, houve uma predominância de estudos qualitativos 53,33\% (08 artigos) em relação aos estudos quantitativos 20\% (03 artigos) e quanti-qualitativos 26,67 (04 artigos). Em relação ao delineamento, destacamos que a maioria dos estudos analisados, com 46,67\% (7 artigos), não especificaram o delineamento que seguiram. Dentre os estudos que especificaram, os estudos longitudinais são predominantes representando 20\% (03 artigos).

Em relação à essência do conteúdo e produção dos conhecimentos se destacaram no total dos artigos selecionados, em ordem de percentualidade decrescente: a prática de saúde baseada na concepção de saúde mais abrangente e libertadora com 66,67\% dos 15 estudos (10 artigos); em seguida tivemos: uma visão de saúde ainda centrada no modelo biomédico, focando na higiene e prevenção de doenças com um achado de 53,33\% (8 artigos); a forma utilizada de abordagem do tema saúde na escola também com 53,33\% (8 artigos); a formação do currículo escolar e o Projeto Político Pedagógico obtendo 40\% dos estudos (6 artigos); $26,67 \%$ pontuaram as dificuldades de desenvolver ações de saúde na escola (4 artigos); $20 \%$ ponderaram a importância em trabalhar as temáticas de saúde na escola (3 artigos), e também 20\% a percepção e visão que possuem do termo saúde (03 artigos); 13,33\% citaram a formação dos professores (2 artigos), assim como também o mesmo quantitativo para as seguintes pontuações: preparação dos professores para trabalhar com as temáticas de saúde e da importância da participação dos pais.

A análise crítica quanto à temática, metodologia e resultados dos estudos, possibilitou a construção de 02 (duas) categorias: A formação dos educadores e a repercussão na prática da saúde escolar; e As vertentes educacionais presentes na prática da saúde escolar. Dos 15 (quinze) artigos selecionados, 09 (nove) foram alocados na primeira categoria e 06 (seis) na segunda categoria analítica construída.

\section{DISCUSSÃO}

\section{A formação dos educadores e a repercussão na prática da saúde escolar}

Todos os artigos analisados (100\%) enfatizam a necessidade dos professores e agentes de educação nas escolas, como pais, profissionais da saúde e outros, sentirem-se responsáveis pela educação em saúde em seus ambientes, e da necessidade de conhecimento teórico e técnico para desenvolvimento dessas ações de forma eficaz.

No estudo de Fernandes, Rocha, Souza, (2005), os professores foram questionados sobre o conteúdo do tema 'saúde' em sua formação docente e $22,2 \%$ responderam não terem recebido nenhum tipo de informação sobre o tema. Entre os 77,7\% que afirmaram terem estudado conteúdos relativos á questões de saúde, 34,29\% não consideraram satisfatório os conteúdos ministrados. Destes, 50\% argumentaram que as informações eram superficiais. Portanto, a temática Educação em Saúde Escolar merece ser ressaltada na formação dos profissionais de educação, a fim de prepará-los para desenvolverem tais atividades educativas nas escolas com maior propriedade (Sarreta, 2009).

Ainda em relação à preparação para trabalhar com o tema transversal 'saúde' dentro do ambiente escolar, 40\% dos professores afirmaram que não se sentiam aptos para o desenvolvimento desses conteúdos (Fernandes, Rocha, Souza, 2005). Os profissionais envolvidos no processo de construção de uma cultura saudável nas escolas devem ser incentivados e aprimorados para produzir e desenvolver estraté- 
gias de educação em saúde com os estudantes (Juzwiak, Castro, Batista, 2013).

Em relação ao tema "Educação em saúde na escola", 20\% dos artigos (03 artigos) revelam que há educadores interessados em estimular os alunos em relação aos hábitos de vida saudáveis, concentrando-se nessa temática. Os autores referem que é preciso informar os estudantes e incentivar o desenvolvimento de habilidades que contribuam para conscientização e consequente reorientação de hábitos (Santos, Bógus, 2007). Juzwiak, Castro, Batista, (2013) sugerem algumas estratégias de aprendizado tais como: espaço de reflexão e crítica que favoreçam a integração e ideias e a reorientação de suas práticas de saúde.

Discutir questões relativas à educação saúde com os alunos é relevante na opinião dos docentes. Desse modo, 20\% das publicações (03 artigos) citaram essa questão. Em uma pesquisa, os professores ao serem indagados sobre a prática da saúde escolar, 20\% citaram a necessidade de conhecer e prevenir doenças, $15 \%$ consideraram importante conhecer noções de higiene e alimentação, 15\% o preparo e capacitação dos alunos para cuidar de sua saúde, 8,33\% privilegiam trabalhar questões relativas a cidadania e a qualidade de vida (Fernandes, Rocha, Souza, 2005). Ressalta-se que a maioria dos docentes, relaciona o tema à prevenção de doenças, não levando em conta as demandas dos educandos e a promoção da saúde.

Quanto à forma de abordagem de temas sobre educação em saúde, verificou-se que $53,33 \%$ (08 artigos), se referiram à essa questão. Notou-se nas respostas que, mesmo sabendo de que tais temas devem ser abordados em todas as disciplinas, prevaleceu a visão de que os responsáveis pelos temas de educação em saúde são apenas os professores de Ciên- cias. Além disso, alguns professores atribuem as ações programáticas de educação em saúde, assim como o processo de planejamento, à autonomia do diretor e do coordenador pedagógico, não entendendo que todos os professores devem participar de todo o processo assim como os próprios alunos (Santos, Bógus, 2007; Leonello, L’Abbate, 2006).

Observou-se também em 46,67\% dos estudos (07 artigos) a importância do envolvimento da comunidade e de outros profissionais na prática educativa em saúde escolar. Juzwiak, Castro, Batista, (2013) relataram em estudo que a interação, troca de experiências e conhecimento promovidas durante as oficinas realizadas na escola estimulou a participação e o trabalho em parceria. Maciel, Oliveira, Frechiani, Sales, Brotto, Araújo, (2010); Antunes, Antunes, Corvino, (2008) consideram indispensável a integração dos serviços de saúde para a solidificação de uma atividade intersetorial e interdisciplinar, favorecendo atividades de promoção da saúde.

Foi enfatizado em 13,33\% dos estudos (02 artigos) que é indispensável a participação de pais e cuidadores, considerando a adequação, satisfação e contexto cultural nas intervenções com estudantes e suas famílias (Fernández, Dinamarca, Bercovich, Vio, 2013; Casemiro, Fonseca, Secco, 2014). Nos depoimentos, os pais mostraram-se motivados a promover a saúde por meio de hábitos saudáveis, envolvendo os filhos e outros familiares (Santos, Caetano, Moreira, 2011).

Outra pesquisa observou a correlação positiva existente entre o número de encontros com a comunidade escolar para abordar a temática alimentação saudável e a presença de ambientes saudáveis. As escolas que realizavam encontros frequentes para abordar o assunto alimentação saudável possuíam, em 
menor proporção, ambulantes e/ou comércios locais próximos. Tal fato revela que a aproximação da escola com o contexto em que está inserida, estimula mudanças importantes e significativas no âmbito das práticas de educação em saúde (Silva, Schmitz, Rodrigues, Gabril, 2013).

Dentre os estudos analisados, 26,67\% (04 artigos) abordavam as dificuldades encontradas pelos professores para o desenvolvimento das práticas educativas em saúde no âmbito da saúde escolar. Um artigo ressaltou que professores mal remunerados se referem às ações de Educação em Saúde como um "trabalho extra" (Kain, Uauy, Concha, Leyton, Bustos, Salazar, Lobos, Vio, 2012). Outro estudo, fez referência às dificuldades em trabalhar o tema 'saúde' no ambiente escolar, (40\%) dos participantes referiram-se à falta de material didático, (20\%) à falta de capacitação específica, (15\%) à falta de apoio do ambiente familiar, e (10\%) à falta de recursos humanos (Fernandes, Rocha, Souza, 2005).

Cabe ressaltar que $40 \%$ dos estudos (6 artigos) destacaram as dificuldades relacionadas ao currículo escolar e o Projeto Político Pedagógico (PPP). Outros estudos apontam que o planejamento e execução do Programa de Saúde Escolar tem sido difícil tarefa para os professores devido ao tempo de para cumprimento do currículo. Na maioria das vezes, os currículos são inflexíveis e disponibilizam pouco tempo para o desenvolvimento das práticas educativas em saúde (Kain, Uauy, Concha, Leyton, Bustos, Salazar, Lobos, Vio, 2012; Leonello, L’Abbate, 2006).

Uma outra pesquisa realizada relata que o PPP de uma escola que inclui temas relativos à saúde, não deixou claro a transversalidade e nem a interdisciplinaridade inerentes á saúde escolar. Mais uma vez destacou-se que o

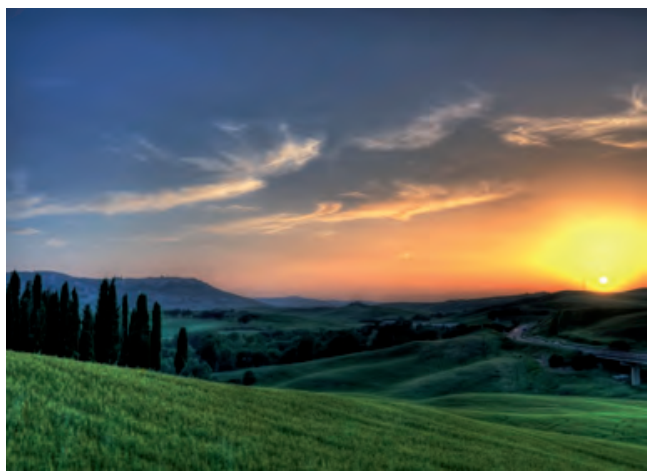

conteúdo dessa temática foi abordado principalmente pela disciplina de Ciências (Cunha, Sousa, Machado, 2010). Outro artigo selecionado relata que a integração de ações de educação em saúde no currículo permitiu abordar esta temática na sala de aula, servindo como uma ligação entre a escola e a comunidade (Antunes, Antunes, Corvino, 2008).

Uma informação importante descrita em um estudo dá conta que a maioria das escolas que possuía parceria com os profissionais de saúde e incluía apenas o tema "alimentação saudável” no currículo escolar (Silva, Schmitz, Rodrigues, Gabril, 2013). Percebe-se então, o quanto ainda é preciso estimular a intersetorialidade e incentivar o desenvolvimento das práticas educativas em saúde nas escolas.

\section{As vertentes educacionais presentes na práti-} ca da saúde escolar

Após a análise dos estudos, observou-se que os professores continuam, na maioria das vezes, destacando questões relacionadas à higiene, alimentação e prevenção de doenças. Tal fato foi exemplificado em 53,33\% dos estudos analisados (08 artigos). Esta realidade reflete a reprodução do paradigma biomédico, que prioriza o indivíduo e a doença, em detrimento da coletividade e da promoção da saúde. Por outro lado, 33,34\% dos estudos (05 
artigos) revelaram que professores desenvolveram uma articulação importante entre as questões de qualidade de vida e de cidadania (Fernandes, Rocha, Souza, 2005).

Embora o discurso atual no domínio da educação enfatize a tendência libertadora, a prática pedagógica predominante nas escolas tem evidenciado a continuidade da transmissão de conhecimentos desarticulado da realidade social e da experiência de vida do discente. Isso precisa ser modificado o quanto antes para garantir a execução e sucesso das práticas educativas de promoção em saúde no âmbito da saúde escolar (Santos, Vidal, Bittencourt, Boery, Sena, 2011).

No contexto das práticas de educação em saúde baseadas na concepção de saúde libertadora e participativa, Fernández, Dinamarca, Bercovich, Vio (2013) destaca a necessidade de novas linhas metodológicas de pesquisa avaliativas. Tais pesquisas deverão considerar fatores comportamentais, características pessoais e influências ambientais que permitam favorecer as práticas de educação em saúde no âmbito da promoção na escola para crianças.

Segundo os sujeitos do estudo de Santos, Penna, Moriyama, Leão, Souza, Aguiar, (2009) as estratégias utilizadas na prática da educação em saúde promoveram a participação, a troca de conhecimentos e o diálogo entre os envolvidos. Considerou-se a realidade local, levando-se em conta os aspectos socioeconômicos e culturais, como a literatura recomenda. Acreditando-se que o trabalho educativo em saúde deve criar condições para que os partícipes construam um novo conhecimento. Essa vivência contribuiu para mudanças, as quais foram percebidas na prática educativa dos educadores e nas atitudes dos educandos.

Um outro estudo selecionado destaca a utilização de estratégias que levam em conta o contexto e interesse dos educandos com a implementação de dinâmicas de integração e comunicação, atividades de dança, exposições dialogadas, diferentes recursos audiovisuais, estudos dirigidos por meio de leitura de textos/artigos e relatos de experiências. Além disso, foram utilizados fóruns on line para a integração e troca de informações entre os participantes durante os intervalos entre as oficinas. Percebeu-se no estudo que a adoção de estratégias variadas e participativas no processo de educação em saúde na escola favoreceram o planejamento de novas atividades, a redefinição de temas, metodologias e estratégias, indispensáveis para o sucesso da intervenção (Juzwiak, Castro, Batista, 2013).

A tendência pedagógica libertadora entende o discente como centro do processo educacional, devendo ser considerado o seu contexto sociocultural. Apoiado na perspectiva freiriana, estabelece a concepção de educar para a decisão, para a responsabilidade social e política, trazendo à tona a contextualização do discente na educação como ator e não mais como sujeito (Freire, 2000).

Acredita-se que as estratégias de educação em saúde para enfrentar esta problemática encontram na Educação Libertadora um forte embasamento teórico e pedagógico a ser delineado a partir das relações do homem com a realidade, por meio dos quais os diferentes atores podem se tornar os autores de suas escolhas e responsável por seus próprios caminhos (Freire, 2000).

Para isso, faz-se necessário superar a visão fragmentada do conhecer e agir em saúde e de um saber científico descontextualizado. Com isso, integrar o saber científico ao saber popular para construção e reconstrução do conhecimento tem mostrado ser o caminho mais ade $\neg$ quado e eficaz para alcançar os efeitos 
almejados (Araújo, Araújo, Souza, Simpson, 2011; Antunes, Antunes, Corvino, 2008).

\section{CONCLUSÃO}

Os achados desta pesquisa permitiram conhecer o que está sendo produzido pela comunidade científica sobre práticas educativas no âmbito da saúde escolar, no período entre 2005 até 2014. Foi possível perceber que tal prática ainda necessita aprimoramento nas ações dos educadores no Programa de Saúde Escolar.

Torna-se necessário a realização de atividades educação permanente favorecendo uma maior articulação entre os profissionais da área da saúde e da educação. A saúde escolar precisa ser mais discutida com os docentes, em todos os segmentos da escola, com os pais e com a comunidade, pois ainda não se sabe de fato o significado dessa prática educativa em saúde. Vale ressaltar que é essencial conhecer a realidade vivenciada pelos escolares, no intuito de gerar reflexões sobre a temática, promovendo a intersetorialidade entre a saúde e a educação, constituindo-se como uma estratégia fundamental para a ocorrência desse processo, especialmente por meio de metodologias ativas.

Portanto, esse campo do conhecimento em saúde precisa ser refletido e levado em consideração por todos os atores sociais envolvidos. Além disso, os gestores da área de educação precisam compreender que os profissionais necessitam de condições para a realização das ações de educação em saúde além da capacitação.

Conclui-se que são necessárias ações educativas voltadas para a realidade de cada comunidade, que considerem os diversos aspectos, as influências ambientais e os fatores metodológicos, além das características comportamentais e pessoais que visam melhorias na qualidade de vida e saúde, gerando uma repercussão positiva na saúde dos escolares.

\section{REFERÊNCIAS}

- Antunes, L.S., Antunes, L.A.A., \& Corvino, M.P. (2008). Educative practices and attitudes within the pre-school environment: evaluating the education professionals. Brazilian Oral Research, 22(4), 340-5.

- Araújo, L.M. de., Araújo, L.M. de., Souza, E.C.R. de., \& Simpson, C.A. (2011). Condições de saúde de escolares e intervenção de enfermagem: relato de experiência. Revista Rene, Fortaleza, 12(4), 841-8.

- Balbino, C.M. (2010). A gerência do cuidado de enfermagem na implantação de um espaço de cuidar em saúde à comunidade escolar. (Dissertação). Universidade Federal Fluminense. Rio de Janeiro, Niterói.

- Casemiro, J.P., Fonseca, A.B.C. da., \& Secco, F.V.M. (2014). Promover saúde na escola: reflexões a partir de uma revisão sobre saúde escolar na América Latina. Ciência \& Saúde Coletiva, 19 (3), 829-840.

- Cunha, E. da., Sousa, A.A. de., \& Machado, N.M.V. (2010). A alimentação orgânica e as ações educativas na escola: diagnóstico para a educação em saúde e nutrição. Ciência \& Saúde Coletiva, 15(1), 39-49.

- Fernandes, M.H., Rocha, V.M., \& Souza, D.B. de. (2005). A concepção sobre saúde do escolar entre professores do ensino fundamental ( $1^{\mathrm{a}}$ a $4^{\mathrm{a}}$ séries). História, Ciências, Saúde-Manguinhos, v. 12, n. 2, 283-91.

- Fernández, L.L.L., Dinamarca, B.L., Bercovich, J.K., \& Vio, F. (2013). Evaluación de una intervención educativa para la prevención de la obesidad infantil en escuelas básicas de Chile. Nutr Hosp, 28(4), 1156-1164.

- Figueiredo, T.A.M. de., Machado, V.L.T., \& Abreu, M.M.S. de. (2010). A saúde na escola: um breve resgate histórico. Ciência \& Saúde Coletiva, 15(2), 397-402.

- Freire, P. (2000). Educação como prática da liberdade. 24.ed. Rio de Janeiro: Paz e Terra.

- Juzwiak, C.R., Castro, P.M. de., \& Batista, S.H.S. da. S. (2013). A experiência da Oficina Permanente de Educação Alimentar e em Saúde (OPEAS): formação de profissionais para a promoção da alimentação saudável nas escolas. Ciência \& Saúde Coletiva, 18(4), 1009-1018.

- Kain, J., Uauy, R., Concha, F., Leyton, B., Bustos, N., Salazar, G., Lobos, L., \& Vio, F. (2012). School-Based Obesity 
Prevention Interventions for Chilean Children During the Past Decades: Lessons Learned. American Society for Nutrition, 3, 616-621.

- Leonello, V.M., \& L’Abbate, S. (2006). Educação em Saúde na escola: uma abordagem do currículo e da percepção de alunos de graduação em Pedagogia. Interface Comunicação, Saúde, Educação, v.9, n.18, 149-66.

- Maciel, E.L.N., Oliveira, C.B., Frechiani, J.M., Sales, C.M.M., Brotto, L.D. de. A., \& Araújo, M. D. (2010). Projeto Aprendendo Saúde na Escola: a experiência de repercussões positivas na qualidade de vida e determinantes da saúde de membros de uma comunidade escolar em Vitória, Espírito Santo. Ciência \& Saúde Coletiva, 15(2), 389-396.

- Martins, M.E.C., \& Brito, I.S. (2013). Investigação-ação participativa em saúde: revisão integrativa da literatura em língua portuguesa. (Dissertação) Universidade de Coimbra, Portugal.

- Maté, E.R., \& Maté, E.M. (2015). Primeras intervenciones higiénico - sanitarias en el ámbito escolar: el caso de Navarra. Cultura de los Cuidados (Edição digital), 19(43). Disponível em: http://dx.doi. org/10.14198/ cuid.2015.43.07.

- Mendes, K.D.S., Silveira, R.C.C.P., \& Galvão, C.M. (2008). Revisão integrativa: método de pesquisa para a incorporação de evidências na saúde e na enfermagem.
Texto \& Contexto - Enfermagem. 17(4), 758-764.

- Santos, F.P. dos. A., Vidal, L.M., Bittencourt, I.S., Boery, R.N.S. de. O., \& Sena, E.L. da. S. (2011). Estratégias de enfrentamento dos dilemas bioéticos gerados pela violencia na escola. Physis Revista de Saúde Coletiva, 21(1), 267-281.

- Santos, I.G. dos., Penna, C.L., Moriyama, F.M., Leão, F.V., Souza, M.P.D. de., \& Aguiar, Z.N. (2009). Percepção dos educadores e coordenadores de uma creche sobre processo educativo em saúde desenvolvido com abordagem multiprofissional. Rev. APS, 12(4), 409-419.

- Santos, K.F. dos., \& Bógus, C.M. (2007). A percepção de educadores sobre a escola promotora de saúde: um estudo de caso. Rev Bras Crescimento Desenvolv Hum, 17(3), 123-133.

- Santos, Z.M.S.A., Caetano, J.A., \& Moreira, F.G.A. (2011). Atuação dos pais na prevenção da hipertensão arterial - uma tecnologia educativa em saúde. Ciência \& Saúde Coletiva, 16(11), 4385-4394.

- Sarreta, F.O. (2009) Educação permanente em saúde para os trabalhadores do SUS. São Paulo: Cultura Acadêmica.

- Silva, J.R.M., Schmitz, B. de. A.S., Rodrigues, M. de. L.C.F., \& Gabriel, C.G. (2013). Promotion of healthy eating at schools in the Federal District of Brazil. Rev. Nutr., Campinas, 26(2), 145-158.

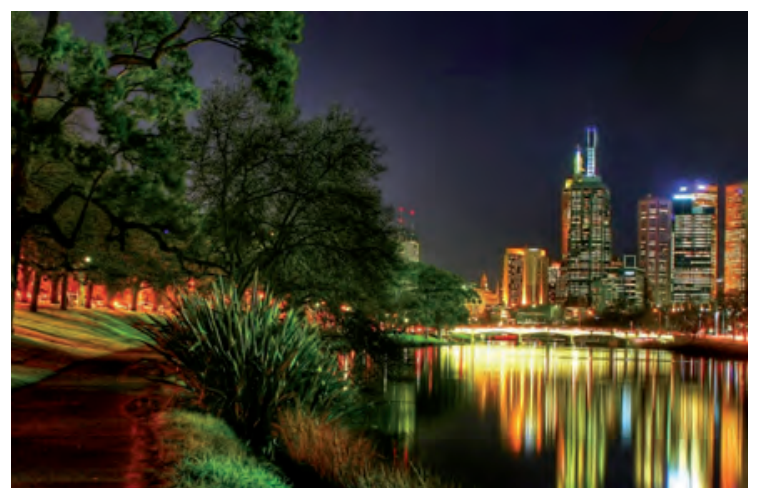

